\title{
The influence of stimulus dimensionality and viewing orientation on detection of symmetry in dot patterns
}

\author{
PAUL LOCHER \\ Montclair State College, Upper Montclair, New Jersey \\ and \\ GERDA SMETS \\ Delft University of Technology, Delft, The Netherlands
}

\begin{abstract}
We tested whether depth and visual motion contribute to detection of the global percept of mirror symmetry in briefly exposed masked dot patterns viewed orthogonally and nonorthogonally. Subjects discriminated between symmetric and either asymmetric patterns (Task 1) or their perturbed counterparts degraded by the presence of random elements (Task 2). Receiver operating characteristic analysis revealed that neither depth nor motion provided perceptually useful information about a pattern's symmetry beyond that contained in a two-dimensional view, suggesting that these processing modules can be ignored when modeling the perceptual properties of symmetric dot displays. Results also revealed significantly lower levels of detectability for nonorthogonal versus orthogonal regard and for Task 2 versus Task 1 conditions. Performance in the former conditions was, however, well above chance levels, demonstrating that the mechanism responsible for symmetry detection is sufficiently robust to tolerate deviation in feature regularities that characterize visual symmetry.
\end{abstract}

The influence of local and global attributes of symmetric patterns on the perceptibility of the global percept of mirror symmetry was recently investigated by Locher and Wagemans (1990). They found that a highly salient percept of symmetry emerged when pattern elements were clustered together within a pattern or grouped in symmetric pairs along a single symmetry axis or two orthogonal axes. Detectability of symmetry was not, however, systematically influenced by type of component pattern element (i.e., dots, line segments in different orientations, or a mixture of line-segment orientations). Locher and Wagemans concluded that mirror symmetry is detected preattentively by some kind of integral code that emerges at the earliest stage of visual processing from the interaction of pattern elements and the way they are organized spatially. They proposed that symmetry is a salient regularity of a visual display that is automatically coded and signaled by spatial grouping processes responsible for construction of the full primal sketch.

As with the many other symmetry-detection studies, Locher and Wagemans's (1990) subjects responded to

This research was completed while the first author was Visiting Professor in the Department of Form Theory at the Delft University of Technology. This version of the paper profits from the comments of K. Overbeeke, C. Nodine, E. Krupinski, and J. Wagemans. We thank E. Krupinski for performing the signal detection analyses. Requests for reprints should be sent to either Paul Locher, Department of Psychology, Montclair State College, Upper Montclair, NJ 07043, or Gerda Smets, Faculteit van het Industrieel Ontwerpen, Technische Universiteit Delft, Jaffalaan 9, 2628 BX Delft, The Netherlands. "static" two-dimensional displays that were orthogonal to the line of sight. Experience with symmetric patterns outside the laboratory, however, typically involves threedimensional stimuli that are seen "at an angle" and frequently in motion. It remains to be determined, however, whether these factors contribute perceptually useful information to the salience of the global percept of symmetry. The present study was a first step in addressing this issue.

To examine the effects of increased dimensionality on detectability of global symmetry, dot patterns studied by Locher and Wagemans (1990) were seen by subjects in the present experiment under two-, three-, and fourdimensional conditions. Stimulus dots were arranged on a two-dimensional (2-D) plane or in a three-dimensional (3-D) volume. In the third stimulus situation, subjects viewed the 3-D stimuli as they rotated about the vertical axis. This temporal property makes the condition fourdimensional (4-D) in a "space-time" context, that is, stimuli have three spatial coordinates and one temporal one.

How might stereoscopic disparity and correspondence information provided by the third dimension and by motion be expected to influence symmetry detection? There is a considerable body of neurophysiological and perceptual evidence that the visual system is organized at the earliest stages such that the analyses of different properties (e.g., form, depth, motion) proceed largely independently of each other (see, e.g., Wagemans, 1988, for an overview of this evidence).

If processing modules for different properties are autonomous at the lowest levels of vision, and if symmetry 
is a perceptual primitive whose presence is coded and signaled automatically by primal sketch processes as proposed by Locher and Wagemans (1990), then the salience of bilateral symmetry in dot patterns viewed orthogonally should not depend on the output of depth and motion processing modules. Binocular disparity and correspondence information would provide little additional evidence of the regularities needed to signal the presence of symmetry. Jenkins (1983) has shown that these regularities include midpoint collinearity (i.e., midpoints of the virtual lines connecting correlated place tokens relative to the axis of reflection are situated on a straight line) and orientational uniformity (i.e., virtual lines connecting symmetric elements all have the same orientation). Thus, increased dimensionality would not be expected to enhance detection of symmetry in either static or dynamic 3-D patterns over that for 2-D patterns viewed orthogonally.

When symmetric patterns are viewed nonorthogonally, retinal image distortions, if sufficiently large, of feature regularities characteristic of symmetry should result in the detection mechanism producing a less-than-optimal signal. If this is the case, then reduced symmetry detection would be expected for patterns viewed nonorthogonally versus the same patterns viewed with orthogonal regard. This hypothesis was tested in the present study by comparing detection performance when symmetric patterns were seen at an angle of $0^{\circ}$ versus $22.5^{\circ}$ to the line of sight. Selection of the angle for the "skewed" viewing condition was based on results of an investigation by Wagemans, Van Gool, and d'Ydewalle (1990) of the influence of skewness (among other variables) in symmetric dot patterns viewed orthogonally on visual search processes. They found that element-pair comparisons took more time and resulted in more errors when they were oriented at angles between the main orientations (e.g., $22.5^{\circ}$ vs. $0^{\circ}$ and $45^{\circ}$ ).

Although disparity and correspondence information were not expected to enhance symmetry detection in orthogonal viewing conditions, they may provide cues to the existence of higher order invariant relations among elements in skewed patterns, thereby enhancing the percept of symmetry. Such cues might include regularities in virtual disparity lines or affinities between paired correspondence tokens (see Ullman, 1979). If this is the case, the global pattern of stimulus information produced by depth and motion might supplement and help reveal the presence of symmetry as compared with a static view of the 2-D version of the same stimulus. Such a finding would demonstrate that other, yet unknown, regularities signaling the presence of bilateral symmetry must be added to those identified by Jenkins (1983). It would also suggest that symmetry detection involves more than primal sketch processes as hypothesized by Locher and Wagemans (1990).

In summary, a rating-task signal detection procedure was used in the present study to examine the combined influence of stimulus dimensionality (2-D, 3-D, and 4-D) and viewing orientation $\left(0^{\circ}\right.$ and $\left.22.5^{\circ}\right)$ on symmetry-detection performance. In addition, a third factor (task) was also included to investigate whether the mechanism responsible for symmetry detection is sufficiently robust under the conditions outlined above to signal the presence of pattern elements not fitting in the global percept of symmetry. Subjects discriminated between symmetric and asymmetric patterns (Task 1) and between the same symmetric patterns and their perturbations, which were composed of two-thirds symmetric and one-third asymmetric element pairs (Task 2).

\section{METHOD}

\section{Subjects}

Six members of the Department of Form Theory at the Delft University of Technology volunteered as subjects.

\section{Stimuli and Apparatus}

The one asymmetric, four symmetric, and four perturbed dot patterns used in this research were selected from the set of stimuli studied by Locher and Wagemans (1990). Details concerning the patterns' construction can be found in their paper. Each pattern consisted of 36 dots spaced evenly in an imaginary $13 \times 13$ grid. In the three single-axis symmetric stimuli, 18 dots on one side of each pattern were mirror reflected on the opposite side of that pattern. These patterns contained either 3, 4, or 5 element pairs in the region surrounding the symmetry axis. A doubleaxis pattern, the fourth symmetric stimulus, consisted of nine dots in the upper left quadrant mirrored about the horizontal and vertical axes.

A perturbed version of each symmetric stimulus was generated by repositioning six dots throughout the pattern to grid locations above, below, to the left of, to the right of, and diagonal to their locations in its symmetric counterpart. Symmetric patterns and their perturbations are shown in Figure 1.

The asymmetric stimulus was composed of dots not occupying mirror grid locations about either the horizontal or vertical midline. In addition, dot versions of Locher and Wagemans's (1990) mask displays were used in the present research.

Each 2-D stimulus was constructed on an $18-\mathrm{cm}^{2}$ piece of heavy cardboard painted matte black. The 36 matte white dots ( $2 \mathrm{~mm}$ in diameter) of each pattern occupied a $6.5 \times 6.5 \mathrm{~cm}$ area in the center of the cardboard. At the viewing distance of $95 \mathrm{~cm}$, each pattern subtended $3.9^{\circ}$ horizontally and vertically and each dot subtended $7.25^{\prime}$ of arc. The 3-D version of each pattern, which also subtended $3.9^{\circ}$ horizontally and vertically, consisted of 36 white beads ( $2 \mathrm{~mm}$ in diameter) mounted on wires projecting from an $18-\mathrm{cm}^{2}$ piece of sheet metal (see Figure 2). Symmetric and perturbed dot pairs were located on one of seven planes within the volumetric stimulus space (see Figure 3), which subtended $5^{\prime}$ of arc in depth $(2 \mathrm{~cm})$ when viewed at the same distance as 2-D stimuli. All axis element pairs were located on the central plane (Plane 4 in Figure 3), which appeared at the same distance from the eye as the planar surface of the 2-D stimuli. No vergence aid other than the stimuli them-

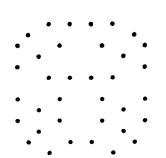

A1

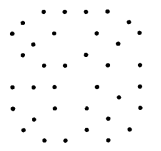

B1

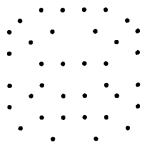

A2

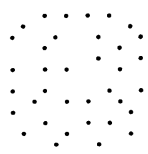

B2

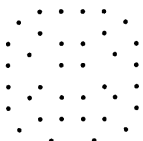

A3

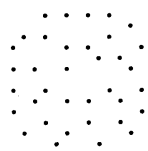

B3

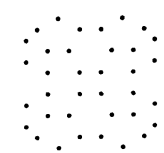

A4
Figure 1. The four symmetric patterns are shown in Row 1. Columns 1, 2, and 3 contain patterns with 3,4 , and 5 element pairs in the region surrounding the single symmetry axis oriented vertically. The pattern in Column 4 is symmetric about the horizontal and vertical axes. Row 2 contains the corresponding perturbed version of each symmetric pattern in Row 1. 
selves and feedback concerning response accuracy was used. Background surfaces and dots of all stimuli and masks were prepared with the same nonreflective black or white paints (Talons Co. TALETA products) to ensure the same luminance of all displays.

The apparatus consisted of a Gerbrands three-field tachistoscope (Model T-3B-2) with a solid object cabinet (Type G1151) fitted to the port of the second field. Presentation time was controlled by a fourchannel electronic digital timer (Model 300-4L). Stimuli were held in a motor-driven frame in the center of the solid object cabinet.

In static 2-D and 3-D conditions, stimuli were either positioned in a frontoparallel projective plane (i.e., vertical and orthogonal to the line of sight) or rotated $22.5^{\circ}$ around the vertical axis (see Figure 2). In the 3-D motion conditions, stimuli were rotated continuously about the vertical axis and illuminated through $15^{\circ}$ of angular displacement. For the orthogonal and nonorthogonal viewing conditions, this displacement extended from $+7.5^{\circ}$ to $-7.5^{\circ}$ and $-15^{\circ}$ to $-30^{\circ}$, respectively $\left(0^{\circ}=\right.$ vertical frontoparallel orientation as shown in Figure 2).

Evidence exists that the visual displays and presentation time used in this research are adequate for the establishment of the perception of depth (e.g., Uttal, 1983) and the extraction of a coherent 3-D structure from moving objects (e.g., Lappin, Doner, \& Kottas, 1980).

\section{Procedure}

Each trial began with the signal "ready" spoken by the experimenter, followed immediately by the 130 -msec stimulus presentation, and with its cessation, a masking field that remained on the screen for $500 \mathrm{msec}$. Locher and Wagemans (1990) determined that this exposure duration gave an average error rate of approximately $75 \%$, the performance criterion chosen for the present study. The subject responded either "symmetric" or "asymmetric" (or "perturbed" in Task 2) to each stimulus and indicated the degree of confidence (low, medium, high) in his/her response. The subjects were informed of the accuracy of each response.

Following practice trials involving all stimulus presentation conditions, each subject completed 36 blocks of trials constituting all combinations of the factors dimension, viewing orientation, task, and a fourth three-level factor-symmetry axis orientation-not previously mentioned. This factor reflects the fact that within a block of trials, the axis of symmetry was oriented about either the vertical (V), horizontal $(\mathrm{H})$, or both (B) of these axes. Prior to the start of a block of trials, the subjects were told the orientation of the patterns' axes and instructed to respond "symmetric" when they perceived symmetry about that axis or axes in Condition B. Although Locher and Wagemans (1990) found no consistent effect of axis orientation on detection performance for the stimuli studied

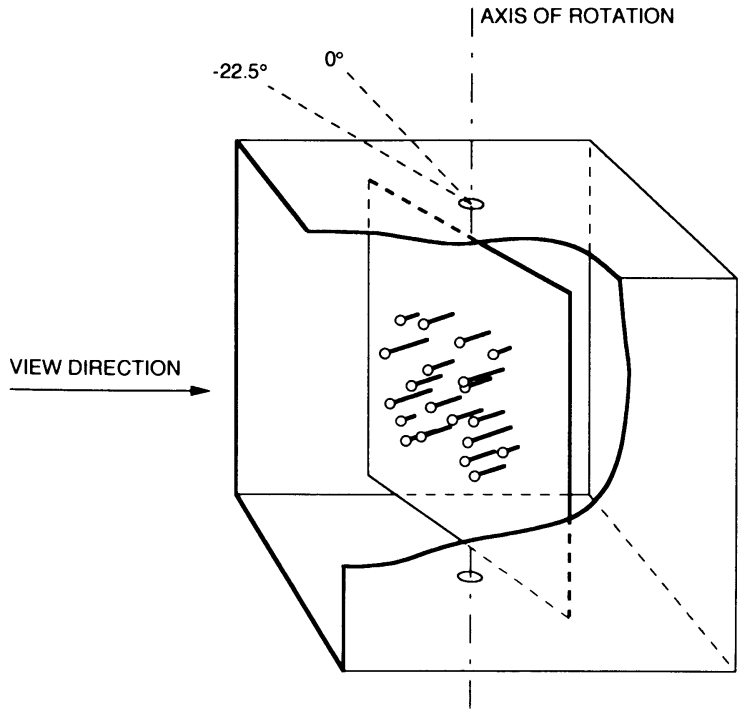

Figure 2. The figure depicts a 3-D stimulus held in the solid object cabinet of the tachistoscope. In the 2-D and 3-D static conditions, stimuli were positioned either orthogonally $\left(0^{\circ}\right)$ or nonorthogonally $\left(-22.5^{\circ}\right)$ to the line of sight as depicted in the figure.

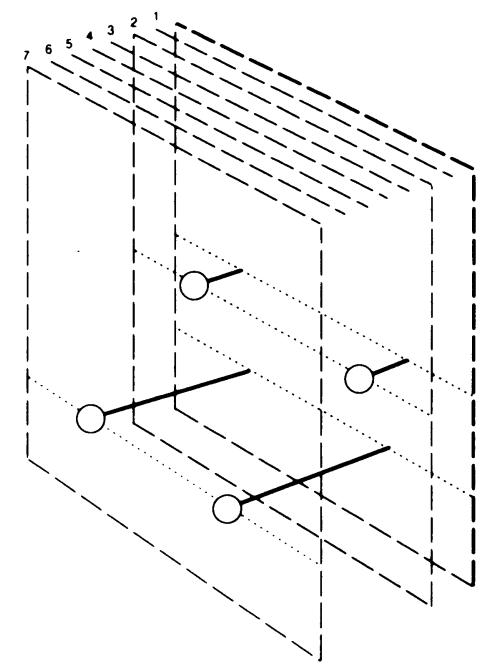

Figure 3. Diagram of the volumetric 3-D stimulus space showing the seven planes at which correlated element pairs were located. Only two element pairs are shown for purposes of this illustration, one pair at Plane 7 and a second pair at Plane 2.

here, research on the influence of axis orientation on the salience of symmetry is equivocal. Thus, manipulation of axis orientation sought to control for this potentially confounding variable.

Tasks were counterbalanced across subjects; all other factors and the order of the 16 stimuli in each block were completely randomized for each subject. Each block consisted of the four symmetric stimuli, each seen in two orientations determined by the $\mathrm{V}, \mathrm{H}$, and $\mathrm{B}$ axis orientation conditions, that is, $0^{\circ}$ and $180^{\circ}, 90^{\circ}$ and $270^{\circ}$, or $0^{\circ}$ and $90^{\circ}$, respectively. In addition, each block contained either the asymmetric pattern seen twice in each of its four orientations or the four perturbed patterns also seen in two orientations determined by the axis orientation condition. Each subject completed 576 individual trials in two sessions, each lasting approximately $2 \mathrm{~h}$.

\section{RESULTS}

Area under the receiver operating characteristic curve values $(A z)$ was used as the index of detection accuracy (see Hanley \& McNeil, 1982). Preliminary analyses revealed no significant difference in detection accuracy due to axis orientation or type of symmetric pattern. Therefore, data were pooled across these factors before calculating $A z$ values, which were then analyzed with a 3 (dimension) $\times 2$ (viewing orientation) $\times 2$ (task) within-subject analysis of variance. The data obtained are summarized in Table 1.

The analysis revealed a significant main effect for dimensions $[F(2,10)=6.95, p<.01]$. A post hoc Tukey test showed that the 4-D condition resulted in a significant $(p<.05)$ reduction in area scores as compared with performance with 2-D patterns $(M s=0.804$ vs. 0.865$)$. Detection accuracy for the 3-D condition $(M=0.845)$ did not reliably differ from that for the 2-D and 4-D conditions.

Also significant were the main effects for viewing orientation $[F(1,5)=53.70, p<.001]$ and task $[F(1,5)=$ $10.68, p<.02]$. Detection accuracy was reduced, on average, from 0.874 with orthogonal regard to 0.801 when patterns were nonorthogonal to the line of sight. And area scores were higher when the stimulus set contained sym- 
Table 1

Mean ROC Area Scores (Az) and Standard Deviations as a Function of Viewing Orientation, Task, and Dimension

\begin{tabular}{|c|c|c|c|c|c|c|c|}
\hline \multirow{3}{*}{$\begin{array}{l}\text { Viewing } \\
\text { Orientation }\end{array}$} & \multirow[b]{3}{*}{ Task } & \multicolumn{6}{|c|}{ Dimension } \\
\hline & & \multicolumn{2}{|c|}{ 2-D } & \multicolumn{2}{|c|}{ 3-D } & \multicolumn{2}{|c|}{ 4-D } \\
\hline & & $M$ & $S D$ & $M$ & $S D$ & $M$ & $S D$ \\
\hline \multirow[t]{2}{*}{ Orthogonal } & S vs. A & .938 & .066 & .948 & .045 & .872 & .081 \\
\hline & S vs. $P$ & .826 & .044 & .848 & .096 & .814 & .050 \\
\hline \multirow[t]{2}{*}{ Nonorthogonal } & S vs. A & .887 & .059 & .827 & .064 & .819 & .041 \\
\hline & $\mathrm{S}$ vs. $\mathrm{P}$ & .808 & .051 & .755 & .062 & .712 & .138 \\
\hline
\end{tabular}

Note-S vs. $A=$ symmetric versus asymmetric patterns; $S$ vs. $P=$ symmetric versus perturbed patterns.

metric and asymmetric versus perturbed patterns $(M \mathrm{~s}=$ 0.882 vs. 0.794$)$. None of the interactions were significant.

\section{DISCUSSION}

The finding that there was virtually no effect on detectability of global symmetry in dot patterns provided by the third dimension for either viewing orientation is in accord with results of a series of investigations of form detection in random dot stereograms conducted by Uttal (1983). The general finding of his research was that little global interaction takes place among the dots of a form when stimuli are generated from random dot arrays. Thus, Uttal concluded that "there is relatively weak global interaction among neural mechanisms at different locations to enhance depth perception. To the contrary, whatever depth processing structures exist probably operate more or less independently to encode depth of individual regions but do not substantially reinforce the overall response strength by collective interaction" (p. 114).

If Uttal's (1983) assertion is correct, it follows that detection of symmetry in 2-D and 3-D versions of the same patterns should be similar, as was found in the present research. This is because the third dimension would do little to establish the existence of global spatial regularities in symmetric dot displays beyond those identified by 2-D processes. An important limitation of this conclusion and the present research is suggested by Uttal's finding in the same series of experiments that the visual system is sensitive to the global arrangement of stimulus dots when 3-D forms are defined by dotted outlines rather than by random dot arrays. Thus, the potential advantage forthcoming from depth for symmetry detection must be assessed using both 2-D and 3-D closed contour patterns of various types. And detection of symmetry in everyday, complex, multidimensional visual stimuli must be investigated as well before a definitive conclusion concerning the contribution of increased dimensionality to symmetry detection can be reached.

With respect to the contribution of motion to symmetry detection, it was found that motion of 3-D stimuli produced a significant, although relatively small, reduction in detection accuracy as compared with static presentations of the same stimuli. One interpretation of this unexpected finding is that motion resulted in competitive interference between neighboring dots in successive views. Dot patterns are more "fluid" in structure and more subject to alternate spatial groupings than, for example, patterns composed of line segments. Perhaps different views resulted in "ambiguity" on the part of the detection mechanism concerning which dots correspond to which others in successive views.

Supporting this speculation is the finding by Lappin et al. (1980) that when dot positions of patterns were perfectly correlated in two frames simulating $5.6^{\circ}$ of rotation about the vertical axis, stable perceptual organization of the pattern resulted. However, when the correlation of dot positions was reduced slightly to .97 , perception of a stable organization was considerably reduced. Thus, it is possible that ambiguities in spatial organization resulting from successive views of symmetric dot patterns in the present experiment resulted in a less-than-optimal signal being generated by the detection mechanism. Ullman's (1984) incremental rigidity scheme suggests that the visual system may require a more extended time period than that provided to reach an accurate perception of the symmetric structure of a visual stimulus in motion.
As mentioned, nonorthogonal viewing produced a significant overall reduction in symmetry detection as compared with orthogonal regard. It appears, therefore, that whatever retinal image distortions occurred because of skewing in the regularities that constitute the invariant structure perceived as symmetry, they were sufficient to reduce detectability of the global percept. At the same time, it is important to note that area scores for nonorthogonal viewing remained relatively high as compared with scores for comparable orthogonal regard conditions (see Table 1). This demonstrates that the mechanism responsible for symmetry detection is sufficiently robust to tolerate some degree of deviation in feature regularities that characterize symmetry.

Additional evidence of the mechanism's flexibility is provided by the finding that area scores for Task 2 conditions (symmetry vs. perturbed) were well above chance levels. Apparently, the visual system is able to signal the presence of symmetric patterns degraded by asymmetry, and observers can respond appropriately to the resulting signal. Barlow and Reeves (1979) also observed that symmetric dot patterns viewed orthogonally for $100 \mathrm{msec}$ were still correctly classified (vs. random stimuli) when dots on the left side of symmetric patterns were displaced from their exact mirror positions by a distance up to one tenth that of the total pattern width. Taken together, these findings indicate that symmetry is represented in the visual system as a graded rather than a discrete all-or-nothing property and that the visual system is sensitive to the presence of "imperfect" symmetry.

\section{REFERENCES}

Barlow, H., \& Reeves, B. (1979). The versatility and absolute efficiency of detecting mirror symmetry in random dot displays. Vision Research, 19, 783-793.

HANLEY, J., \& MCNeIL, B. (1982). The meaning and use of the area under a receiver operating characteristic (ROC) curve. Diagnostic Radiology, 143, 29-36.

JENKINS, B. (1983). Component processes in the perception of bilaterally symmetric dot textures. Perception \& Psychophysics, 34, 433-440.

LAPPIN, J., Doner, J., \& KotTas, B. (1980). Minimal conditions for the visual detection of structure and motion in three dimensions. Science, 209, 717-719.

Locher, P., \& Wagemans, J. (1990, November). Effects of element type and spatial grouping on symmetry detection. Paper presented at the 31st Annual Meeting of the Psychonomic Society, New Orleans.

Ullman, S. (1979). The interpretation of visual motion. Cambridge, MA: MIT Press.

UlLman, S. (1984). Maximizing rigidity: The incremental recovery of 3-D structure from rigid and nonrigid motion. Perception, 13, 255-274.

UTTAL, W. (1983). Visual form detection in 3-dimensional space. Hillsdale, NJ: Erlbaum.

WaGEMANS, J. (1988). Modules in vision: A case study of interdisciplinarity in cognitive science. Acta Psychologica, 67, 59-93.

WaGemans, J., VAN Gool, L., \& D'YDEWALLE, G. (1990). Visual search in dot-patterns with bilateral and skewed symmetry. In D. Brogan (Ed.), Visual search (pp. 99-114). London, UK: Taylor \& Francis.

(Manuscript received July 12, 1991.) 